\title{
WHITE DWARF - ACCRETION DISK BOUNDARY LAYERS
}

\author{
Oded Regev \\ Department of Astronomy, Columbia University \\ New York, N.Y. 10027 \\ on leave from: \\ Department of Physics, Technion \\ Israel Institute of Technology, Haifa 32000
}

\begin{abstract}
The method of matched asymptotic expansions is used to calculate the detailed structure of white dwarf- accretion disk boundary layers. Both optically thick and thin boundary layers are investigated for various values of the relevant parameters, as long as the layer's vertical extension remains very much smaller than the white dwarf's radius. Detailed, self consistent solutions for the boundary layer's and disk's structure are calculated and used to find the amount of energy' radiated onto the accreting white dwarf (recently recognized to be an important factor in nova outburst modelling).
\end{abstract}

\section{INTRODUCTION}

The importance of the accretion disk inner boundary layer (BL) is well established. However, since the pioneering work of Lynden-Bell \& Pringle (1974), progress in trying to understand the BL structure has been rather limited. For a recent review, see Shaviv (1987). In cataclysmic variables the problem is particularly important. For example, it is clear that various observational characteristics of dwarf novae (in particular the X-ray emission) have to do with the BL (Pringle, 1977; Pringle \& Savonije, 1979; Tylenda, 1981; Patterson \& Raymond, 1985). Very recently it has been pointed out that the effect of BL heating on the pre-nova accreting white dwarf is of paramount importance in modelling the outburst (Shaviv \& Starrfield, 1987).

Regev (1983) and Regev \& Hougerat (1988) introduced the method of matched asymptotic expansions as a practical tool for obtaining the BL structure self consistently, and demonstrated its viability for the study of optically thin and thick BL's in cataclysmic variables. In this work I would like to report on the results of an extensive parameter space study in which self consistent BL solutions have been found. The parameters include (in addtion to the white dwarf's mass and radius) the angular velocity $-\Omega_{*}$ at the star's surface, $\dot{M}$ - the mass accretion rate and, in certain cases, the pressure (or the temperature) at the $\mathrm{BL}$ inner edge. It has to be pointed out that our method fails whenever some of the basic assumptions break down or another viscosity prescription in the BL is used. This may result in different BL structures with transition regions (Papaloizou \& Stanley, 1986). 


\section{OUTLINE OF THE METHOD}

I describe here only the essentials of the method. A full derivation is given in Regev (1983), hereafter R, for the optically thick case and in Regev \& Hougerat (1988), hereafter RH, for optically thin BLs. Consider the $\mathbf{r}$ component (in cylindrical coordinates) of the momentum equation. In its non-dimensional form, assuming steady state and axial symmetry it is:

$$
\epsilon^{2} u \frac{\partial u}{\partial r}+\epsilon v \frac{\partial u}{\partial z}-\Omega^{2} r=-\epsilon^{2} \frac{1}{\rho} \frac{\partial p}{\partial r}-\frac{1}{r^{2}}+\epsilon^{2} \frac{z^{2}}{r^{4}}
$$

see $\mathrm{R}$ eq. 7 . The symbols here have their usual meaning. Note the role of the small parameter, $\epsilon$, defined to be $\tilde{v}_{s} / \Omega_{K *} R_{*} . \quad \Omega_{K *} \equiv\left(G M_{*} / R_{*}^{3}\right)^{1 / 2}$ is the Keplerian angular velocity at the star's radius, $R_{*}$, and is used to scale $\Omega . \quad \tilde{v}_{s}$ is the sound velocity at a typical point. If $\epsilon \ll 1$, one can expand all the functions in $\epsilon$, and the lowest order approximation should be satisfactory. Dropping terms of order $\epsilon$ and smaller in equation 1 one gets:

$$
\Omega^{2}=\frac{1}{r^{3}}
$$

which means that the Keplerian angular velocity is the lowest order solution. However, one now faces the obvious difficulty of being unable to satisfy the inner boundary condition for $\Omega$ which should be $\Omega_{*}$ and not 1 (Keplerian at $r=R_{*}$ in our units). Obviously, very near the boundary, in the BL, the above outer solution is invalid. The solution may be something of the type used in the so-called standard theory (Shakura \& Sunyaev, 1973), but the price is the loss of consistency and the necessity to treat the BL somehow, separately. It is also quite disturbing that in such a treatment the disk structure does not depend at all on the inner boundary condition.

The remedy is to use boundary layer methods, i.e. to use another expansion (the inner expansion) very near the inner boundary. In order to do this the coordinates (here the $r$-coordinate) have to be stretched, i.e. one defines a new coordinate $r=1+\delta R$ with $\delta(\epsilon)$ very small. Changing variables in eq. $1, r$ to $R$, one gets a possible meaningful equation in lowest order only for a certain choice of $\delta$ as a function of $\epsilon$. This is called the significant limit and it determines essentially the $\mathrm{BL}$ width. For details, see R and RH, as well as Bender \& Orszag (1978) or Van Dyke (1964). While the basis for understanding the method can be found in these last two references, our case is considerably more complicated as it involves several equations in two space variables. We eliminate the $z$-dependence by vertically averaging and using the assumption that the disk, as well as the BL is geometrically thin. We then proceed as explained in R (or RH) when the BL is optically thick and dominated by gas pressure and free-free opacity (optically thin and dominated by radiation pressure and electron scattering opacity).

The decision which assumption to use is based on the following consideration. For each choice of the parameters (notably $\Omega_{*}$, and $\dot{M}$ ) we look for an inner solution and try to match it to the outer solution (letting $\epsilon \rightarrow 0$ ). If matching is impossible, something must be wrong with the assumptions. If we can match, we check our solution for consistency - i.e. if the BL is still geometrically thin and if its optical depth is according to the assumption. In doing this we use ratios of relevant timescales and optical depth formulae similar to the ones of Pringle \& Savonije (1979) and King \& Shaviv (1984).

The outer solution we find is, of course, identical to the Shakura \& Sunyaev (1973) solution. However we improve on it by trying to determine all integration constants from matching with the inner solution and not by new assumptions and approximations. Consider, in particular, the 
angular momentum equation for the outer solution (eq. 17 in R). After integrating on $z$ one gets the familiarly looking equation for the specific angular momentum transport caused by the gradient of visous stresses:

$$
\dot{M} \frac{d}{d r}\left(r^{2} \Omega\right)=-\frac{\alpha}{\mu} \frac{d}{d r}\left(\nu \Sigma r^{3} \frac{d \Omega}{d r}\right)
$$

See eq. 22 in R. This equation is again in nondimensional units. $\alpha$ and $\mu$ are constants, $\nu$ is the viscosity and $\Sigma$ the surface density. Integrating, one needs a constant (call it $C$ ):

$$
\dot{M}\left[\left(r^{2} \Omega\right)-C\right]=-\frac{\alpha}{\mu} \nu \Sigma r^{3} \frac{d \Omega}{d r}
$$

Obviously, $C$ has to do with the ability of the BL to take up angular momentum. See the discussion in Katz (1987) (p. 199) on this matter. The assumption of $C=1$ (see e.g. Pringle, 1981) allows one to decouple the disk structure from the $\mathrm{BL}$ in the manner explained above. Our method, however allows one to determine $C$ from the matching (if at all possible) and construct a consistent approximation by composing it from the outer and inner expansions. In this way we obtain the structure of the accretion disk all the way down to the star. In additon various integral quantities depend on $C$. For example the disk luminosity is $L_{d}=L_{\text {acc }}\left(\frac{3}{2}-C\right)$ and the BL luminosity is $L_{\mathrm{BL}}=L_{\mathrm{acc}}\left[C-\frac{1}{2}\left(1+\Omega_{*}^{2}\right)\right]$ giving together the total luminosity $L_{T}=L_{\mathrm{acc}}\left(1-\frac{1}{2} \Omega_{*}^{2}\right)$, where $L_{\mathrm{acc}} \equiv G M_{*} \dot{M} / R_{*}$.

\section{RESULTS}

The optically thick BL differs from the optically thin one. Let us call them Case A and B respectively. We find that $\delta=\epsilon^{2}$ in Case $\mathrm{A}$, and $\delta=\epsilon$ in Case $\mathrm{B}$. It follows that the BL thickness (in the $r$ direction) is $\sim \epsilon H_{*}$ in Case $\mathrm{A}$ and $\sim H_{*}$ in Case $\mathrm{B}$, where $H_{*}$ is the vertical extension of the BL. This supports the finding of Pringle \& Savonije (1979) who based their argument on a somewhat different consideration.

For all the values of the relevant parameters for which a Case A solution exists and is consistent, we find values of $0.7 \gtrsim C \gtrsim 1.0$. In this case one has to solve a set of four coupled ODE's for the structure of the BL. The equations are given in R (eqs. 34-37). $C$ is found from the matching provided the values of $p$ (or $T$ ) and $\Omega$ are given on the surface. We use a numerical code for the solution of this boundary value problem, which allows to determine $C$ as an eigenvalue. A relaxation method using quasilinearization (Henyey method) is quite effective if a good initial guess is known. Figure 1a is a typical example of Case A. In addition to the obvious departure from the standard solution (dashed curve) one sees also the effect of the BL on the disk up to $r \sim 2 R_{*}$. This result is for $M_{*}=1 M_{\odot}, \quad R_{*}=9 \times 10^{8} \mathrm{~cm}, \Omega_{*}=0.3 \Omega_{K *}, \dot{M}=5.5 \times 10^{-9} M_{\odot} / \mathrm{yr}$. The value of $C$ for this model is found to be $C=0.71$.

In case $\mathrm{B}$, as described in $\mathrm{RH}$, we were able to find an analytical solution for the $\mathrm{BL}$ which can be matched to the outer solution if $C=1$. Thus, the standard solution continues inward until it is smoothly matched with the BL solution. As expected, one gets in this case a much hotter BL (see figure $1 b$ ). This result is for the same white dwarf parameters but for a significantly lower value of $\dot{M}: \dot{M}=3.5 \times 10^{-10} M_{\odot} / \mathrm{yr}$. 

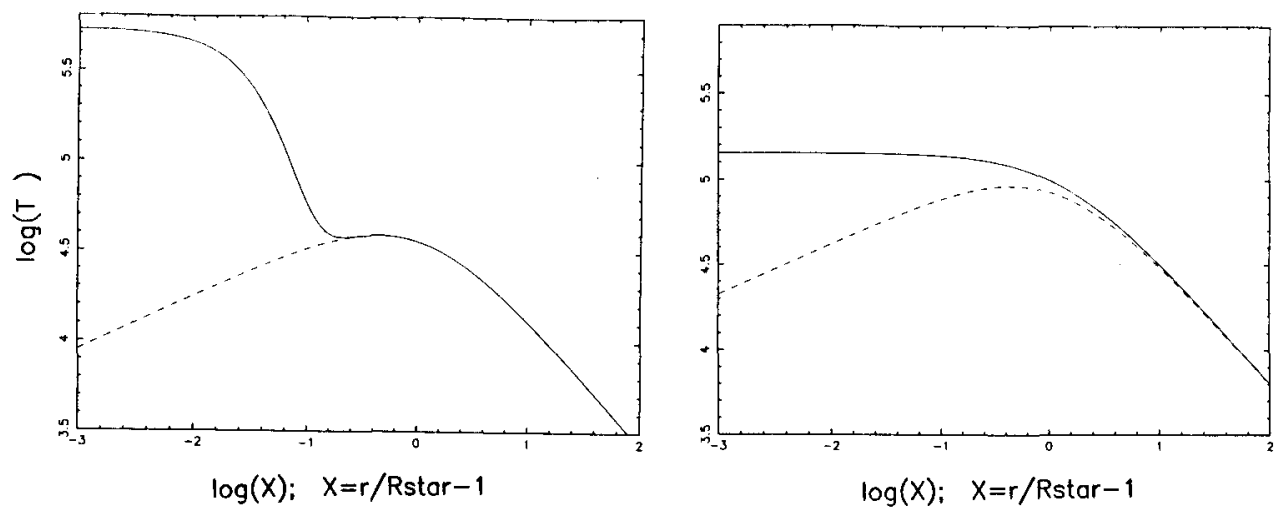

Figure 1: $\log (T)$ (absolute temperature) as a function of position (distance from the white dwarf's edge) in the BL and the inner disk. The right panel (a) is Case A (optically thick) and the left one (b) is Case B (optically thin). The dashed curves are the standard (Shakura \& Sunyaev) solutions for both cases.

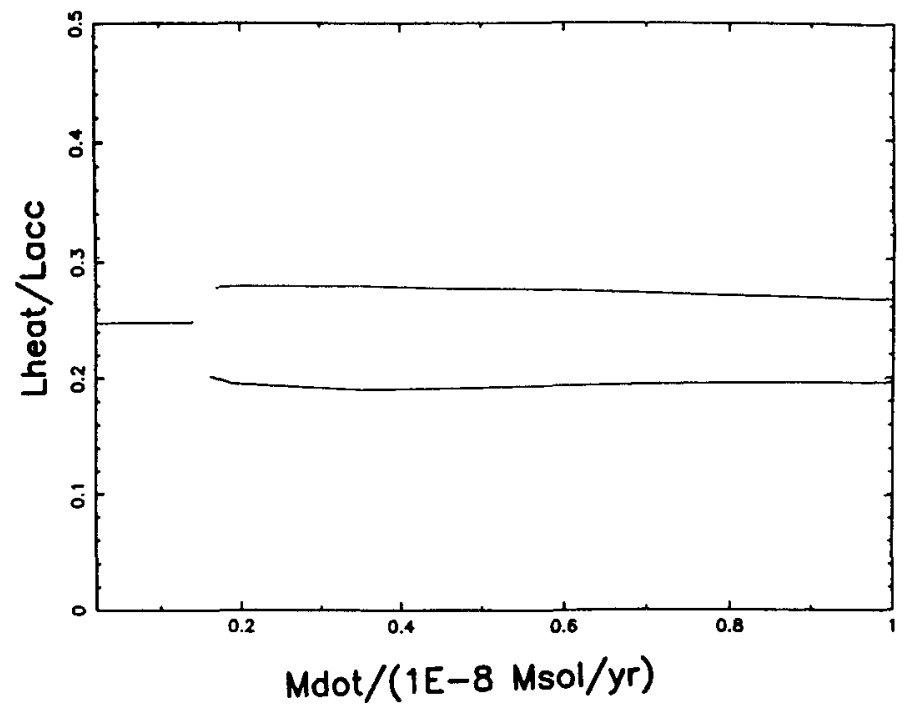

Figure 2: The heating of the white dwarf $\left(L_{\text {heat }}\right)$ in units of $L_{\text {acc }} \equiv G M_{*} \dot{M} / R_{*}$ as a function of $\dot{M}$. The single curve on the left is in the case of an optically thin BL. The two curves on the right delineate the region for which optically thick BL solutions exist.

Finally, I present the results for the amount of radiation falling onto the white dwarf from the $\mathrm{BL}$ and the accretion disk. The effect of this heating on the accreting star and its implications for the nova outburst have recently been studied by Shaviv \& Starrfield (1987). They point out that this heating can significantly affect the evolution to a thermonuclear runaway. The inclusion of this energy makes it more difficult to produce a nova outburst; specifically when $L_{\text {heat }} \sim 0.25 L_{\text {acc }}$, 
the outburst is significantly less violent, and little or no mass is ejected.

Figure 2 depicts the amount of heating of the white dwarf for the same $1 M_{\odot}$ star as before (with $R_{*}=9 \times 10^{8} \mathrm{~cm}$ and $\Omega_{*}=0.3 \Omega_{K_{*}}$ ) as a function of $\dot{M}$. We calculate $L_{\text {heat }}$ by adding the energy radiated into the star (calculated numerically in Case A) to the fraction of the disk and $\mathrm{BL}$ radiation which after being emitted from the surface falls on the WD. In the optically thin case a unique solution of $\alpha \equiv L_{\text {heat }} / L_{\text {acc }}$ equal to 0.248 is obtained. In the optically thick case an addtional free parameter ( the temperature or pressure at the BL-white dwarf boundary) is needed. All the possible values of such a parameter which give a consistent matched solution fall between the two curves on the right side of figure 2. The temperature is lower for the upper limit curve (since then the gradient of $T$ into the star is bigger and hence more energy is radiated inward).

I would like to thank Joe Patterson, Mike Shara and Giora Shaviv for discussions; Norman Baker for letting me use his computer code and the Astronomy Department at Columbia and the Space Telescope Science Institute (where a part of this work has been done) for their hospitality.

\section{REFERENCES}

Bender, C.M. \& Orszag, S.A., 1978, Advanced Methods for Scientists and Engineers, McGraw-Hill. Katz, J.I., 1987, High Energy Astrophsyics, Addison-Wesley.

King, A.R. \& Shaviv, G., 1984, Nature, 308, 519.

Lynden-Bell \& Pringle, J.E., 1974, M.N.R.A.S., 168, 603.

Papaloizou, J.C.B. \& Stanley, C.Q.G., 1986, M.N.R.A.S., 220, 593.

Patterson, J., \& Raymond, J.C., 1985, Ap.J., 292, 535 and 550.

Pringle, J.E., 1977, M.N.R.A.S., 178, 195.

Pringle, J.E., 1981, Ann. Rev. Astr. Ap., 19, 137.

Pringle, J.E. \& Savonije, G.J., 1979, M.N.R.A.S, 198, 777.

Regev, O., 1983, Astr. Ap., 126, 146 (R).

Regev, O. \& Hougerat, A.A., 1988, M.N.R.A.S., 232, 81 (RH).

Shakura, N.I. \& Sunyaev, R.A., 1973, Astr. Ap., 24, 337.

Shaviv, G., 1987, Ap. Sp. Sci., 130, 303.

Shaviv, G. \& Starrfield, S., 1987, Ap.J., 321, L51.

Tylenda, R., 1981, Acta Astr., 31, 127 and 267.

Van Dyke, M., 1964, Perturbation Methods in Fluid Mechanics, Academic Press. 